\title{
Non-Markovian Dynamics of Quantum Discord
}

\author{
F. F. Fanchini, ${ }^{1},{ }^{\text {T T. Werlang, }}{ }^{2}$ C. A. Brasil,${ }^{3}$ L. G. E. Arruda,${ }^{3}$ and A. O. Caldeira ${ }^{1}$ \\ ${ }^{1}$ Instituto de Física Gleb Wataghin, Universidade Estadual de Campinas, \\ P.O. Box 6165, 13083-970, Campinas, SP, Brazil \\ ${ }^{2}$ Departamento de Fúsica, Universidade Federal de São Carlos, \\ P.O. Box 676, 13565-905, São Carlos, SP, Brazil \\ ${ }^{3}$ Instituto de Física de São Carlos, Universidade de São Paulo, \\ P.O. Box 369, 13560-970, São Carlos, SP, Brazil
}

(Dated: October 24, 2018)

\begin{abstract}
We evaluate the quantum discord dynamics of two qubits in independent and common nonMarkovian environments. We compare the dynamics of entanglement with that of quantum discord. For independent reservoirs the quantum discord vanishes only at discrete instants whereas the entanglement can disappear during a finite time interval. For a common reservoir quantum discord and entanglement can behave very differently with sudden birth of the former but not of the latter. Furthermore, in this case the quantum discord dynamics presents sudden changes in the derivative of its time evolution which is evidenced by the presence of kinks in its behavior at discrete instants of time.
\end{abstract}

PACS numbers: 03.65.Ud, 03.65.Yz, 03.67.Mn

\section{INTRODUCTION}

Entanglement is a kind of quantum correlation (QC) that has been playing a central role in quantum information and communication theory 1. However there are other nonclassical correlations apart from entanglement 24 4 that can be of great importance to these fields. In order to characterize all nonclassical correlations, Ollivier and Zurek introduced what they called quantum discord 2]. This measure of quantum correlations captures a fundamental feature of classical bipartite states - when the discord is zero the information is locally accessible and can be obtained by distant independent observers without perturbing the bipartite state. Although a vast literature exists on the study of entanglement just recently the other quantum correlations received due attention [412. A motivation for the study of these correlations, for example, is the recent discovery that nonclassical correlations other than entanglement can be responsible for the quantum computational efficiency of deterministic quantum computation with one pure qubit (DQC1) [5, 6]. In this context, the quantum discord could be a new resource for quantum computation.

However, realistic quantum systems are not closed and therefore it is of fundamental importance to study the quantum correlations when the system loses its coherence due to interactions with the environment 13 . The entanglement dynamics in open quantum systems was broadly studied in the literature but not much exists about the effect of the environment on quantum discord 10 12. A peculiar aspect of the entanglement dynamics is the well-known "entanglement sudden death" (ESD) phenomenon [14, 15]. This process described the finite-

*Electronic address: fanchini@ifi.unicamp.br time disentanglement of two parts that interact with either independent [15-17] or common environments [18 20. In a previous work [10] we observed that, even at finite temperatures, under a dissipative Markovian process, the quantum discord is immune to "sudden death".

Despite the term "entanglement sudden death" sounds mysterious, it is important to note that there is no dynamical distinction between separable and entangled states, since the quantum states can, in general, evolve back and forth across the boundary between distinct fulldimensional subsets of the space of the density matrices which contain separable and entangled states.

The studies on the entanglement dynamics, that initially were restricted to Markovian approximations, have recently been extended to consider non-Markovian environments [17, 19 21. In this case, given the memory stored in the environment, some of the initial entanglement that is lost during the dissipative dynamics can return to the qubits. This phenomenon is known as "sudden-birth of entanglement" (SBE), which in the light of what has been said in the previous paragraph should not present much of a surprise to us. Nevertheless a question still remains; what happens to the quantum discord in this situation? Since quantum discord exists even without entanglement, does it present sudden death or even sudden birth? In order to answer these questions we study the quantum discord of two qubits coupled to non-Markovian dissipative environments.

In this paper we evaluate the quantum discord dynamics for a dissipative non-Markovian process. For independent environments, when the qubits are subject to amplitude damping, we show that it only vanishes at discrete instants of time, each within the time interval when the reduced quantum state becomes pure and separable, and, consequently, the entanglement vanishes. For a common reservoir the quantum discord behavior can be very different from that of the entanglement. While 
the entanglement dynamics presents damped oscillations with or without sudden death, the quantum discord is almost always positive and presents isolated kinks (cuspids) at which there is a jump in its derivatives. The latter behavior is at clear variance with what happens to the entanglement.

\section{QUANTUM DISCORD}

Entanglement is not the only measure of quantum correlations and therefore an interesting approach was introduced in [2, 3] to attempt to quantify all the nonclassical correlations present in a system besides entanglement. The defined quantity - the quantum discord - is given by the difference between two expressions of mutual information (MI) extended from classical to quantum system.

The total correlation between two classical systems $\mathcal{A}$ and $\mathcal{B}$, whose state is described by a joint probability distribution $p(\mathcal{A}, \mathcal{B})$, can be obtained by a measure of the $\mathrm{MI}, \mathcal{I}(\mathcal{A}: \mathcal{B})=H(\mathcal{A})+H(\mathcal{B})-H(\mathcal{A}, \mathcal{B})$, where $H(\cdot)$ denotes the Shannon entropy $H(p)=-\sum_{j k} p_{j k} \log _{2} p_{j k}$ 1]. This classical MI can be rewritten as the equivalent expression $\mathcal{J}(\mathcal{A}: \mathcal{B})=H(\mathcal{A})-H(\mathcal{A} \mid \mathcal{B})$ through the Bayes rule [24], where the conditional entropy $H(\mathcal{A} \mid \mathcal{B})$ quantifies the ignorance about the state of $\mathcal{A}$ when one knows the state of $\mathcal{B}$. For a quantum system represented by a bipartite density operator $\rho$, the Shannon entropy functional is replaced by the von Neumann entropy, $S(\rho)=-\operatorname{Tr}\left(\rho \log _{2} \rho\right)$, which is the first quantum extension of the classical MI. We denote it $\mathcal{I}(\rho)$.

Another route to generalizing the classical MI to the quantum case is to use a measurement-based conditional density operator [2]. If we restrict ourselves to projective measurements performed locally only on system $\mathcal{B}$ described by a complete set of orthogonal projectors, $\left\{\Pi_{k}\right\}$, corresponding to outcomes $k$, the quantum state after a measurement changes to $\rho_{k}=$ $\left[\left(\mathbb{I} \otimes \Pi_{k}\right) \rho\left(\mathbb{I} \otimes \Pi_{k}\right)\right] / \operatorname{Tr}\left(\mathbb{I} \otimes \Pi_{k}\right) \rho\left(\mathbb{I} \otimes \Pi_{k}\right)$, where $\mathbb{I}$ is the identity operator for system $\mathcal{A}$. With this conditional density operator, a quantum analogue of the conditional entropy can then be defined as $S\left(\rho \mid\left\{\Pi_{k}\right\}\right)=$ $\sum_{k} p_{k} S\left(\rho_{k}\right)$, and the second quantum extension of the classical MI may be found, $\mathcal{J}\left(\rho \mid\left\{\Pi_{k}\right\}\right)=S\left(\rho^{\mathcal{A}}\right)-$ $S\left(\rho \mid\left\{\Pi_{k}\right\}\right)$. Projective measurements on system $\mathcal{B}$ remove all nonclassical correlations between $\mathcal{A}$ and $\mathcal{B}$, but the value of $\mathcal{J}\left(\rho \mid\left\{\Pi_{k}\right\}\right)$ depends on the choice of $\left\{\Pi_{k}\right\}$. Therefore, to ensure that it captures all classical correlations, we need to maximize $\mathcal{J}$ over all $\left\{\Pi_{k}\right\}$. This quantity, $\mathcal{Q}(\rho)=\sup _{\left\{\Pi_{k}\right\}} \mathcal{J}\left(\rho \mid\left\{\Pi_{k}\right\}\right)$, is interpreted explicitly by Henderson and Vedral [3], as a measure of classical correlations. The quantum discord is then defined as

$$
D(\rho)=\mathcal{I}(\rho)-\mathcal{Q}(\rho),
$$

and provide us with information on the quantum nature of the correlations between two systems, such that it is zero only for states with classical correlations [2, 3] and nonzero for states with quantum correlations. Although quantum discord is equal to the entanglement of formation for pure states, it is not true for mixed states, since some states present finite quantum discord even without entanglement 2].

It is important to note that to calculate the classical correlations one can consider arbitrary POVM measurements as Henderson and Vedral did in [3]. However, for two qubits, which is our case, Hamieh et al. 22, show that the projective measurement is the POVM which maximizes the classical correlations.

\section{A. Analytical expression for quantum discord}

To evaluate the quantum discord dynamics presented in this article we determine an analytical expression for a subclass of the $X$ structured density operator. We consider a density matrix as given by

$$
\rho(t)=\left(\begin{array}{cccc}
a & 0 & 0 & w \\
0 & b & z & 0 \\
0 & z & b & 0 \\
w & 0 & 0 & d
\end{array}\right)
$$

where the coherences are real numbers and the element $\rho_{22}=\rho_{33}$. It is easy to see that for this expression of $\rho(t)$ the condition $S\left(\rho^{\mathcal{A}}\right)=S\left(\rho^{\mathcal{B}}\right)$ is satisfied and therefore the measurement of classical correlations assumes equal values, irrespective of whether the measurement is performed on the subsystem $\mathcal{A}$ or $\mathcal{B}[3]$. To reduce the difficulty to compute the quantum discord we need to be able to maximize the classical correlation $\mathcal{Q}(\rho)$. This can be done analytically if one notes that a general one-qubit projector can be written as a function of two angles, since

$$
\mathcal{Q}(\rho)=S\left(\rho^{\mathcal{A}}\right)-F(\theta, \phi),
$$

where

$F(\theta, \phi)=\inf _{\{\theta, \phi\}}\left[\sum_{k=1,2} p_{k}(\theta, \phi) S\left(\frac{\Pi_{k}^{\mathcal{B}}(\theta, \phi) \rho^{\mathcal{A B}} \Pi_{k}^{\mathcal{B}}(\theta, \phi)}{p_{k}(\theta, \phi)}\right)\right]$,

with $p_{k}(\theta, \phi)=\operatorname{Tr}\left\{\Pi_{k}^{\mathcal{B}}(\theta, \phi) \rho^{\mathcal{A B}} \Pi_{k}^{\mathcal{B}}(\theta, \phi)\right\}$ and the projectors $\Pi_{k}^{\mathcal{B}}(\theta, \phi)=\mathbb{I} \otimes|k\rangle\langle k|(k=1,2)$ defined by the orthogonal states:

$$
\begin{aligned}
& |1\rangle=\cos \theta|\uparrow\rangle+e^{i \phi} \sin \theta|\downarrow\rangle, \\
& |2\rangle=\sin \theta|\uparrow\rangle-e^{i \phi} \cos \theta|\downarrow\rangle .
\end{aligned}
$$

We begin noting some peculiar properties of $F(\theta, \phi)$ when $\rho^{\mathcal{A B}}$ is given by Eq. 22. Given the structure of the density matrix the critical points of $F(\theta, \phi)$, i.e. the set of values of $\theta$ and $\phi$ such that $\frac{\partial F(\theta, \phi)}{\partial \theta}=0$ and $\frac{\partial F(\theta, \phi)}{\partial \phi}=0$, do not depend on the elements of the density matrix. For $\theta=n \frac{\pi}{2}$ with $n \in \mathbb{Z}$ we have a set of critical points and in this case the function $F(\theta, \phi)$ does not depend on the angle $\phi$. Another set is given by $\theta=m \frac{\pi}{4}$ and $\phi=$ 
$n \frac{\pi}{2}$ with $m, n \in \mathbb{Z}$. Thus, with this observation, using the quantum version of the mutual information $\mathcal{I}$ and Eq. (1), it is straightforward to compute an analytical expression for the quantum discord:

$$
D(\rho)=\min \left\{D_{1}, D_{2}\right\}
$$

where

$$
\begin{aligned}
D_{1} & =S\left(\rho^{\mathcal{A}}\right)-S\left(\rho^{\mathcal{A B}}\right)-a \log _{2}\left(\frac{a}{a+b}\right)-b \log _{2}\left(\frac{b}{a+b}\right) \\
& -d \log _{2}\left(\frac{d}{b+d}\right)-b \log _{2}\left(\frac{b}{d+b}\right),
\end{aligned}
$$

and

$D_{2}=S\left(\rho^{\mathcal{A}}\right)-S\left(\rho^{\mathcal{A B}}\right)-\Delta_{+} \log _{2} \Delta_{+}-\Delta_{-} \log _{2} \Delta_{-},(8)$

with $\Delta_{ \pm}=\frac{1}{2}(1 \pm \Gamma)$ and $\Gamma^{2}=(a-d)^{2}+4(|z|+|w|)^{2}$, which has been numerically verified for any density operator with the same structure as in Eq. 22).

\section{EXACT DISSIPATIVE DYNAMICS OF QUANTUM DISCORD}

In this article we study a system whose dynamics is described by the well-known damped Jaynes-Cummings model. We consider two distinct situations, the independent and common environment. In the former, each qubit is coupled to its own reservoir since the dissipative processes occur independently. In the common environment case, on the other hand, we consider only one bath for both qubits. We suppose that the qubits are coupled to a single cavity mode which in turn is coupled to a non-Markovian environment that initially is in the vacuum state. In this case we can say that the interaction Hamiltonian reduces the amplitude of motion of each qubit state, and this justifies why such a process is known in the literature as the amplitude damping channel. The solutions of these simple models have recently been used to study the non-Markovian effects on the dynamics of entanglement [17, 20]. The environments are represented by a bath of harmonic oscillators, and the spectral density is of the form

$$
J(\omega)=\frac{1}{2 \pi} \frac{\gamma_{0} \lambda^{2}}{\left(\omega_{0}-\omega\right)^{2}+\lambda^{2}},
$$

where $\lambda$ is connected to the reservoir correlation time $\tau_{B}$ by the relation $\tau_{B} \approx 1 / \lambda$, and $\gamma_{0}$ is related to the time scale $\tau_{R}$ over which the state of the system changes, $\tau_{R} \approx 1 / \gamma_{0}$. Here we will consider the strong coupling limit, i. e. $\tau_{R}<2 \tau_{B}$.

For independent amplitude damping channels the twoqubit Hamiltonian can be written as

$H=\omega_{0}^{(i)} \sigma_{+}^{(i)} \sigma_{-}^{(i)}+\sum_{k} \omega_{k}^{(i)} a_{k}^{(i)} a_{k}^{(i)}+\left(\sigma_{+}^{(i)} B^{(i)}+\sigma_{-}^{(i)} B^{(i)^{\dagger}}\right)$, where $B^{(i)}=\sum_{k} g_{k}^{(i)} a_{k}^{(i)}$ with $g_{k}^{(i)}$ being the coupling constant, $\omega_{0}^{(i)}$ is the transition frequency of the i-th qubit, and $\sigma_{ \pm}^{(i)}$ are the system raising and lowering operators of the i-th qubit. Here the index $k$ labels the reservoir field modes with frequencies $\omega_{k}^{(i)}$, and $a_{k}^{(i)^{\dagger}}\left(a_{k}^{(i)}\right)$ is their usual creation (annihilation) operator. Here and in the following the Einstein convention sum is adopted.

For common environments, on the other hand, we have that the system raising (lowering) operator of each qubit is coupled to the same environment operator $B\left(B^{\dagger}\right)$. In this case we have one bath coupled to both qubits, and the Hamiltonian is given by

$$
H=\omega_{0}^{(i)} \sigma_{+}^{(i)} \sigma_{-}^{(i)}+\sum_{k} \omega_{k} a_{k}^{\dagger} a_{k}+\left(\sigma_{+}^{(i)} B+\sigma_{-}^{(i)} B^{\dagger}\right) .
$$

We have considered two identical atoms equally coupled to the reservoir. In this case, the dynamics of the two qubits occur in two completely decoupled subspaces, generated by $\{|00\rangle,|+\rangle=(|10\rangle+|01\rangle) / \sqrt{2},|11\rangle\}$ and $\{|-\rangle=(|10\rangle-|01\rangle) / \sqrt{2}\}$. Using this fact, Mazzola et al. [20] connect the problem with a three-level ladder system [25] and through the pseudomode approach [26], the dynamics can be evaluated without any approximations.

The initial state considered in this paper is the Bell-like state:

$$
|\psi\rangle=\alpha|00\rangle+\sqrt{1-\alpha^{2}}|11\rangle,
$$

and thus the density matrix of the atomic system has the form of Eq. 22 with the dynamics of its matrix elements given in [17] for independent reservoir and 20, 23] for common reservoir.

In Fig. (1) we plot the quantum discord as a function of the scaled time $\gamma_{0} t$ in the strong coupling regime, $\lambda=$ $0.1 \gamma_{0}$ for the common reservoir case and $\lambda=0.01 \gamma_{0}$ for independent reservoirs. The initial state used in Fig. (1) is given by Eq. 12 with $\alpha^{2}=1 / 3$. The analytical solution of the quantum discord is the minimum value assumed by the functions $D_{1}$ (squares) and $D_{2}$ (circles), given by Eq. (7) and Eq. (8) respectively. In Fig. (1) it is represented by the black (solid) line.

We have observed that, for the values of $\gamma_{0} t$ indicated by the doted lines in Fig (1), the angles that minimize the discord change, point out to a "sudden change" of discord as previously noted in 11 for Markovian environments. In the region between two consecutive dotted lines the angles remain unchanged. As shown in Fig.1b, the same analysis applies to independent reservoirs, but no "sudden change" is observed in this case. Here it should be emphasized that what we are calling "sudden change of discord" is actually a signature of a jump of the time derivatives of that function at specific instants.

In order to compare the discord dynamics with the entanglement dynamics we used the entanglement of formation (EoF) 27] as a measure of entanglement. For two qubits, the EoF dynamics can be written as a function 

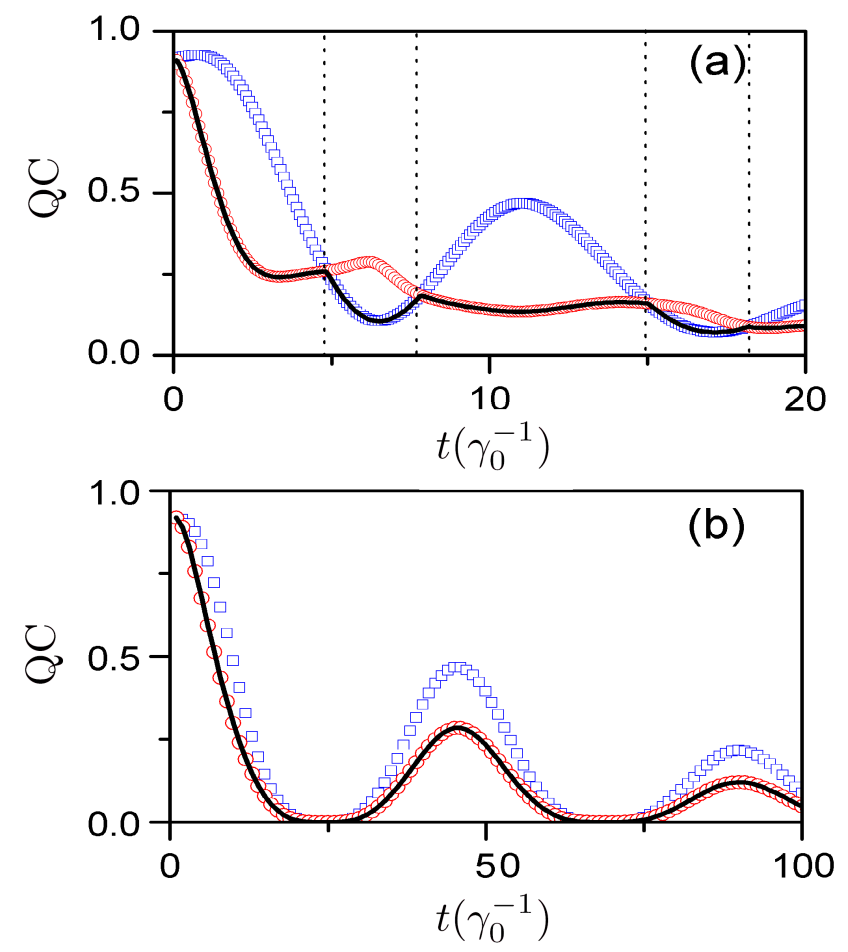

FIG. 1: (Color online) Analytical ( $D_{1}$-squares, and $D_{2}$ circles) and numerical (solid line) dynamics of discord for (a) a common reservoir with $\lambda=0.1 \gamma_{0}$ and (b) independent reservoirs with $\lambda=0.01 \gamma_{0}$. The dotted lines in (a) indicate the value of $\gamma_{0} t$ where the sudden change occurs. The two-qubits initial state used here is given in Eq. 12 with $\alpha^{2}=1 / 3$.

of the concurrence [28] and it is given by

$$
E(t)=-\Gamma(t) \log _{2} \Gamma(t)-[1-\Gamma(t)] \log _{2}[1-\Gamma(t)]
$$

where $\Gamma(t)=\frac{1}{2}\left(1+\sqrt{1-C(t)^{2}}\right)$ with $C(t)$ being the time dependent concurrence. For a density matrix with a structure defined as in Eq. (2) we have that $C(t)=$ $2 \max \left\{0, \Lambda_{1}(\mathrm{t}), \Lambda_{2}(\mathrm{t})\right\}$ with $\Lambda_{1}(t)=z(t)-\sqrt{a(t) d(t)}$ and $\Lambda_{2}(t)=w(t)-b(t)$.

We begin analyzing the entanglement and quantum discord dynamics for independent reservoirs. The entanglement dynamics, for example, shows different behaviors depending on the initial state of the two-qubit system [17]. In the case where $\alpha^{2} \geq 1 / 2$ the EoF periodically vanishes for the discrete times defined by $t_{n}=2[n \pi-\arctan d / \lambda] / d$ with $d=\sqrt{2 \gamma_{0} \lambda-\lambda^{2}}$ and $n$ an integer [17]. This behavior is illustrated in Fig. (2c) (dashed line) where we observe that the amplitude of oscillation undergoes a decay after each revival. However, when $\alpha^{2}>1 / 2$ the behavior of the EoF presents two different features: $(i)$ there is ESD because the EoF permanently vanishes within finite time intervals [see Fig.2a (dashed line)] and ( $i i)$ the revival of entanglement after these intervals when the two qubits are fully disentangled [see Fig.2b (dashed line)].

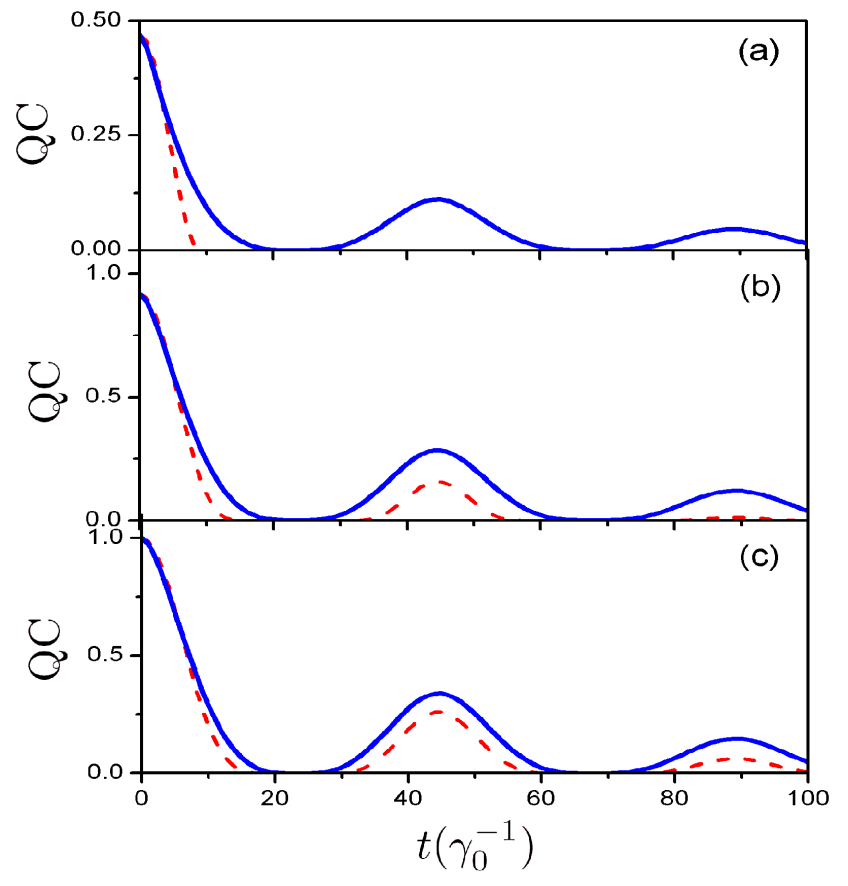

FIG. 2: (Color online) Dynamics of Discord (solid line) and EoF (dashed line) as a function of the scaled time for independent reservoirs with $\lambda=0.01 \gamma_{0}$ and the two qubits initially prepared in the state of eq. 12 with (a) $\alpha^{2}=1 / 10$, (b) $\alpha^{2}=1 / 3$, and (c) $\alpha^{2}=1 / 2$.

The discord dynamics for all initial entangled states $\left(0<\alpha^{2}<1\right)$ is similar to the EoF for $\alpha^{2} \geq 1 / 2$, i.e., the discord vanishes only at $t_{n}$ when the two-qubits state becomes the separable pure state $|00\rangle$ [see Fig.2a-c (solid line)]. The nonclassical correlations are mediated by the reservoir, since there is no interaction between the qubits. Furthermore, whereas the entanglement may reappear after a time interval within which the EoF is zero, the discord is almost always non zero. This result indicates that the discord under non-Markovian dissipative dynamics, likewise the Markovian case, vanishes only at discrete instants. This point agrees with the results presented in [12, where the authors show that the states with zero QD form a set of measure zero.

In the case where the two-qubits interact with the same environment the entanglement dynamics presents two regimes [20]: for $\alpha^{2} \gtrsim 1 / 4$ damped oscillations of entanglement are observed [see Fig.3d, dashed line], and for $\alpha^{2} \lesssim 1 / 4$ finite time intervals of complete disentanglement are followed by entanglement revivals [see Fig.3b-c, dashed line]. However, while for independent reservoirs the discord and entanglement behaviors are similar to each other, for common environment they behave very differently, as show in Fig.3a-d. For $\alpha^{2}=1 / 2$ both the discord and entanglement dynamics present the same behavior, but as $\alpha^{2}$ decreases the discord exhibits very complicated damped oscillations with sudden changes more 


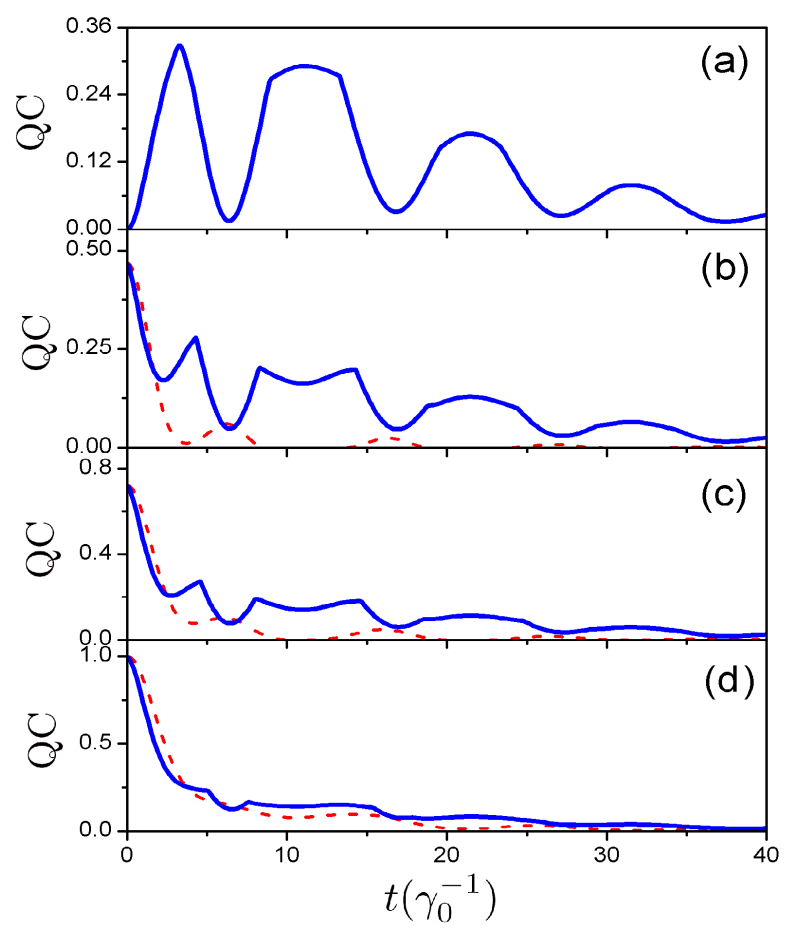

FIG. 3: (Color online) Dynamics of Discord (solid line) and EoF (dashed line) as a function of the scaled time for common reservoirs with $\lambda=0.1 \gamma_{0}$ and two qubits initially prepared in the state of eq. 12 with (a) $\alpha^{2}=0$, (b) $\alpha^{2}=1 / 10$, (c) $\alpha^{2}=1 / 5$, and (d) $\alpha^{2}=1 / 2$.
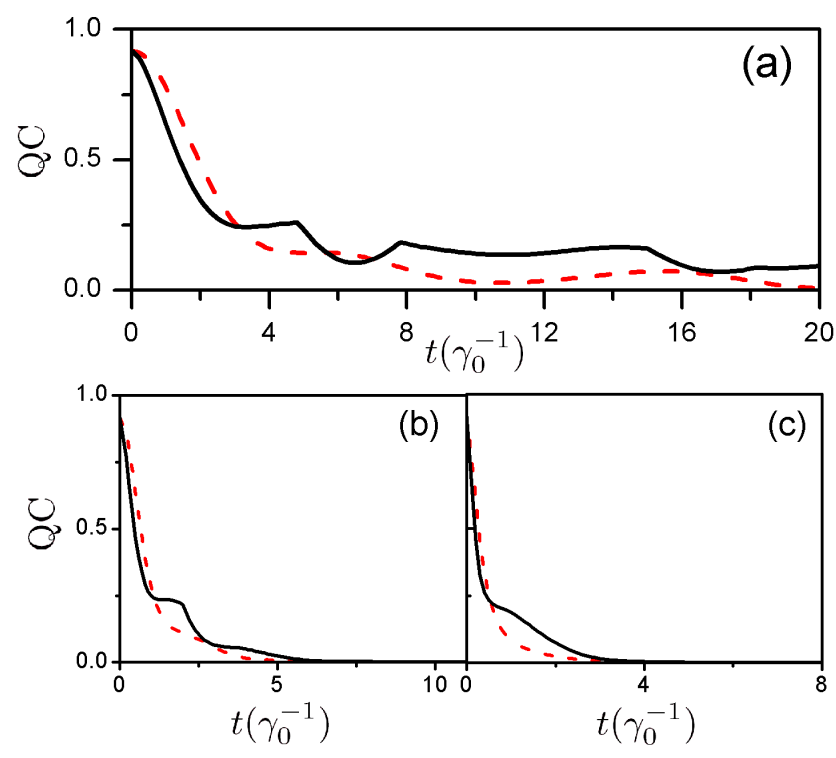

FIG. 4: (Color online) Dynamics of Discord (solid line) and EoF (dashed line) as a function of the scaled time for a common reservoir with (a) $\lambda=0.1 \gamma_{0}$, (b) $\lambda=\gamma_{0}$, and (c) $\lambda=10 \gamma_{0}$. The two qubits initial state is given by eq. 12 with $\alpha^{2}=1 / 3$. evident. The difference between these two measures is even more drastic when $\alpha^{2}=0$, where the two-qubit state is initially the separable state $|11\rangle$. In this case the interaction between the qubits mediated by a common reservoir does not lead to the generation of entanglement between them [20]. On the other hand, the reservoirmediated interaction leads to the generation of nonclassical correlations as exposed in Fig. (3a), showing a "sudden birth" of discord but not of entanglement. Moreover, since the reservoir is initially in the vacuum state, when the initial state is $|00\rangle$, obtained from Eq. (12) with $\alpha^{2}=1$, no correlation is created since the composite system is in its ground state. It is also interesting to note in Fig.3b-c that the decrease of entanglement is accompanied by the increase of discord in some regions.

In Fig. 4 we plotted the discord (solid line) and the EoF (dashed line) as a function of the scaled time $\gamma_{0} t$ for a common reservoir with (a) $\lambda=0.1 \gamma_{0}$, (b) $\lambda=\gamma_{0}$, and (c) $\lambda=10 \gamma_{0}$. The two-qubits are initially in the state (12) with $\alpha^{2}=1 / 3$. These results show that the entanglement decay almost exponentially for $\lambda=10 \gamma_{0}$, as in the case of Markovian reservoirs. It is expected because in this regime $\lambda>2 \gamma_{0}$ and therefore the qubit-reservoir coupling is weak. In this same weak coupling regime the discord has a similar behavior and we note that the number of points where the sudden change occur also tends to decrease. Besides, as the effective coupling between the two qubits is due to the action of the common reservoir, the quantum correlations created tends to decrease.

It is worth mentioning that results very similar to ours, for the case of independent environments, have been numerically studied in [29].

\section{CONCLUSION}

We have studied the quantum discord dynamics of two-qubits coupled to common and independent nonMarkovian environments. We have used the exactly solvable damped Jaynes-Cummings model for zero temperature environments. We have observed that even when the entanglement suddenly disappears and reappears after finite time intervals, the quantum discord vanishes only at discrete times. For a common environment we have observed what is called the sudden change phenomenon. Actually, the quantum discord between the qubits suddenly changes depending on the maximization process of the amount of classical correlations between them. This fact indicates that this phenomenon could be universal which means that, for general initial conditions and interaction Hamiltonians, we expect that the POVM that maximizes the classical correlations would abruptly change from one time interval to the other. This point will be studied in a future work where more general Hamiltonians and initial conditions will be considered. Furthermore, we have observed that, in the case of common environments, the very different behavior of discord and entanglement can arise even for initially separated 
states. Finally, we have also noticed that, even without entanglement, the correlations introduced by the environment are transferred to the two qubits producing a finite quantum discord.

\section{ACKNOWLEDGMENTS}

We wish to thank the partial financial support from the Fundação de Amparo à Pesquisa de São Paulo (FAPESP) and Conselho Nacional de Desenvolvimento Científico Tecnológico $(\mathrm{CNPq})$. We thank Adriana Auyuanet for pointing out a mistake in an early draft. AOC also acknowledges his participation as a member of the Instituto Nacional de Ciência e Tecnologia em Informaçãao Quântica (INCT-IQ).
[1] M. A. Nielsen and I. L. Chuang, Quantum Computation and Quantum Information (Cambridge University Press, Cambridge, England, 2000).

[2] H. Ollivier and W. H. Zurek, Phys. Rev. Lett. 88, 017901 (2001).

[3] L. Henderson and V. Vedral, J. Phys. A 34, 6899 (2001); V. Vedral, Phys. Rev. Lett 90, 050401 (2003).

[4] S. Luo, Phys. Rev. A 77, 042303 (2008).

[5] A. Datta, A. Shaji, and C. M. Caves, Phys. Rev. Lett. 100, 050502 (2008).

[6] B. P. Lanyon, M. Barbieri, M. P. Almeida, and A. G. White, Phys. Rev. Lett. 101, 200501 (2008).

[7] R. Dillenschneider, Phys. Rev. B 78, 224413 (2008); M. S. Sarandy, Phys. Rev. A 80, 022108 (2009).

[8] J. Cui and H. Fan , arXiv:0904.2703v1 (2009).

[9] C. A. Rodriguez-Rosario et al., J. Phys. A: Math. Theor. 41, 205301 (2008); A. Shabani and D. A. Lidar, Phys. Rev. Lett. 102, 100402 (2009).

[10] T. Werlang, S. Souza, F. F. Fanchini, and C. J. VillasBoas, Phys. Rev. A 80, 024103 (2009).

[11] J. Maziero, L. C. Celeri, R. M. Serra, and V. Vedral, Phys. Rev. A 80, 044102 (2009).

[12] A. Ferraro et al., arXiv:0908.3157v2 (2009).

[13] H.-P. Breuer and F. Petruccione, The Theory of Open Quantum Systems (Oxford University Press, Oxford, New York, 2002).

[14] K. Zyczkowski, P. Horodecki, M. Horodecki, and R. Horodecki, Phys. Rev. A 65, 012101 (2001); L. Diósi, Lec. Notes Phys. 622, 157 (2003); P. J. Dodd and J. J. Halliwell, Phys. Rev. A 69, 052105 (2004).

[15] T. Yu and J. H. Eberly, Phys. Rev. Lett. 93, 140404 (2004).

[16] T. Yu and J. H. Eberly, Phys. Rev. Lett. 93, 140404 (2004); M. F. Santos, P. Milman, L. Davidovich, and
N. Zagury, Phys. Rev. A 73, 040305(R) (2006); M. P. Almeida et al., Science 316, 579 (2007); L. Aolita, R. Chaves, D. Cavalcanti, A. Acin, and L. Davidovich, Phys. Rev. Lett. 100, 080501 (2008); A. Salles et al., Phys. Rev. A 78, 022322 (2008); C. E. López, G. Romero, F. Lastra, E. Solano, and J. C. Retamal, Phys. Rev. Lett. 101, 080503 (2008).

[17] B. Bellomo, R. Lo Franco, and G. Compagno, Phys. Rev. Lett. 99, 160502 (2007);

[18] Z. Ficek and R. Tanás, Phys. Rev. A 74, 024304 (2006).

[19] J. P. Paz and A. J. Roncaglia, Phys. Rev. Lett. 100, 220401 (2008).

[20] L. Mazzola, S. Maniscalco, J. Piilo, K. A. Suominen, and B. M. Garraway, Phys. Rev. A 79, 042302 (2009).

[21] J. Dajka, M. Mierzejewski, and J. Luczka, Phys. Rev. A 77, 042316 (2008 ); F. Q. Wang, Z. M. Zhang, and R. S. Liang, Phys. Rev. A 78, 062318 (2008); K. Shiokawa, Phys. Rev. A 79, 012308 (2009).

[22] S. Hamieh, R. Kobes, and H. Zaraket, Phys. Rev. A 70, 052325 (2004).

[23] L. Mazzola, S. Maniscalco, J. Piilo, and K. A. Suominen, arXiv:0904.2857v1 (2009).

[24] T. M. Cover and J. A. Thomas, Elements of Information Theory (Wiley-Interscience, New York, 2006).

[25] B. J. Dalton and B. M. Garraway, Phys. Rev. A 68, 033809 (2003); B. J. Dalton, S. M. Barnett, and B. M. Garraway, Phys. Rev. A 64, 053813 (2001).

[26] B. M. Garraway, Phys. Rev. A 55, 2290 (1997).

[27] C. H. Bennett, D. P. DiVincenzo, J. A. Smolin, and W. K. Wootters, Phys. Rev. A 54, 3824 (1996).

[28] W. K. Wootters, Phys. Rev. Lett. 80, 2245 (1998).

[29] B. Wang, Z. Xu, Z. Chen, and M. Feng, arXiv:0911.1845v1 (2009). 